\title{
Markov modeling for the neurosurgeon: a review of the literature and an introduction to cost-effectiveness research
}

\author{
*Arvin R. Wali, MAS, ${ }^{1}$ Michael G. Brandel, BA, ${ }^{1}$ David R. Santiago-Dieppa, MD, ${ }^{1}$ \\ Robert C. Rennert, MD, ${ }^{1}$ Jeffrey A. Steinberg, MD, ${ }^{1}$ Brian R. Hirshman, MD, PhD, ${ }^{1}$ \\ James D. Murphy, MD, ${ }^{2}$ and Alexander A. Khalessi, MD ${ }^{1}$ \\ 'Department of Neurological Surgery and 'Radiation Medicine and Applied Sciences, University of California, San Diego, \\ California
}

\begin{abstract}
OBJECTIVE Markov modeling is a clinical research technique that allows competing medical strategies to be mathematically assessed in order to identify the optimal allocation of health care resources. The authors present a review of the recently published neurosurgical literature that employs Markov modeling and provide a conceptual framework with which to evaluate, critique, and apply the findings generated from health economics research.
\end{abstract}

METHODS The PubMed online database was searched to identify neurosurgical literature published from January 2010 to December 2017 that had utilized Markov modeling for neurosurgical cost-effectiveness studies. Included articles were then assessed with regard to year of publication, subspecialty of neurosurgery, decision analytical techniques utilized, and source information for model inputs.

RESULTS A total of 55 articles utilizing Markov models were identified across a broad range of neurosurgical subspecialties. Sixty-five percent of the papers were published within the past 3 years alone. The majority of models derived health transition probabilities, health utilities, and cost information from previously published studies or publicly available information. Only $62 \%$ of the studies incorporated indirect costs. Ninety-three percent of the studies performed a 1-way or 2 -way sensitivity analysis, and $67 \%$ performed a probabilistic sensitivity analysis. A review of the conceptual framework of Markov modeling and an explanation of the different terminology and methodology are provided.

CONCLUSIONS As neurosurgeons continue to innovate and identify novel treatment strategies for patients, Markov modeling will allow for better characterization of the impact of these interventions on a patient and societal level. The aim of this work is to equip the neurosurgical readership with the tools to better understand, critique, and apply findings produced from cost-effectiveness research.

https://thejns.org/doi/abs/10.3171/2018.2.FOCUS17805

KEYWORDS cost-effectiveness research; Markov modeling; decision analytics; neurosurgical health services research

$\mathrm{T}$ HE United States spends nearly $18 \%$ of its gross domestic product on health care (approximately $\$ 3.3$ trillion), far more than any of the other 34 countries in the Organisation for Economic Co-operation and Development (OECD), and this gap is widening. ${ }^{5,12} \mathrm{~A}$ number of demographic shifts have overcome the cost-saving effects of certain economic and policy factors, including the increasing proportions of elderly individuals, ${ }^{25}$ obese and unhealthy Americans, ${ }^{9,32}$ and people living with chronic conditions and disability. ${ }^{14,16}$ For these reasons, it remains a priority for clinicians to maintain heightened scrutiny of health care expenditures, greater focus on cost savings, and increased value for research in health economics.

Cost-effectiveness analysis utilizing Markov modeling is an approach to examine the allocation of limited resources to produce optimal health outputs. ${ }^{4,37}$ Cost-effectiveness research is increasingly important for physicians and health care leadership to make policy and applied

ABBREVIATIONS ICER = incremental cost-effectiveness ratio; PSA = probabilistic sensitivity analysis; $Q A L Y=$ quality-adjusted life year; WTP = willingness to pay. SUBMITTED January 2, 2018. ACCEPTED February 21, 2018.

INCLUDE WHEN CITING DOI: 10.3171/2018.2.FOCUS17805.

${ }^{*}$ A.R.W. and M.G.B. contributed equally to this publication. 
clinical decisions ${ }^{37}$ The use of evidence to improve health care quality while lowering costs will continue to guide clinical practice, ${ }^{21}$ and it will be critical for neurosurgeons to maintain a strong understanding of health economics, decision analytics, and the methodology of cost-effectiveness research in order to remain at the forefront of health leadership and innovation.

However, many surgeons remain unfamiliar with costeffectiveness methodology, and thus the ability to produce and interpret this type of research may be limited. ${ }^{31}$ While prior authors have demonstrated the value of costeffectiveness across a wide array of neurosurgical subspecialties, $, 17,28,29,34-36$ we sought to produce an introductory primer of the different analytical methods available within Markov modeling while investigating recent publication trends and research techniques that have been employed in the current literature.

In this study we aimed to introduce Markov modeling and cost-effectiveness methodology to neurosurgeons seeking to gain familiarity with cost-effectiveness techniques. With an increased understanding of Markov modeling and health economics, the neurosurgical readership will be better equipped to critically analyze cost-effectiveness research and apply these findings in a clinical setting.

\section{Methods}

The PubMed online database was searched to identify neurosurgical literature published from January 1, 2010, through December 1, 2017, that had utilized Markov modeling to determine the relative cost-effectiveness of clinical management strategies. The following query was used: (Neurosurgery AND (cost-effectiveness analysis OR Markov Model)). These broad search criteria were used to identify a robust sample of recently published neurosurgical literature.

Articles were included in this analysis if they 1) employed Markov modeling to conduct cost-effectiveness research and 2) were published in the English language. Articles were excluded if they investigated clinical strategies that were not relevant to neurosurgery. Authors (A.R.W. and M.G.B.) independently reviewed the articles yielded from the PubMed database to ensure appropriate selection according to the inclusion and exclusion criteria. After articles were screened by title and abstract, each paper was individually reviewed to identify the following: 1) year of publication, 2) subspecialty of neurosurgical intervention being investigated, 3) commonly utilized decision analytical techniques within Markov modeling, 4) source information for probabilities of achieving certain health outcomes, 5) source information regarding direct and indirect costs associated with interventions and health outcomes, and 6) source information for quality of life metrics regarding health states.

\section{Results \\ Publication Patterns of Recently Published Cost-Effectiveness Literature}

Of the 457 articles initially identified in the PubMed search, 55 articles ultimately met inclusion and exclusion criteria (Fig. 1). Within recent years, more papers utilizing Markov modeling for cost-effectiveness research have been published (Fig. 2). Thirty-six (65\%) of the articles were published within the last 3 years alone. Among the 55 papers, the subspecialty of neurosurgical topics investigated were diverse: spine $16(29 \%)$, vascular $9(16 \%)$, trauma $9(16 \%)$, tumor/oncology $6(11 \%)$, epilepsy $4(7 \%)$, pain $4(7 \%)$, functional neurosurgery $3(5 \%)$, pituitary/endoscopic skull base 2 (4\%), and peripheral nerve 2 (4\%). A review of the technical aspects of Markov modeling, types of model inputs, and their practical use in cost-effectiveness research is provided below.

\section{Review of Markov Models}

In cost-effectiveness research, Markov models are made to analyze competing treatment strategies available to a patient that can change that patient's health state. Markov modeling allows for the quantification of health utility, a metric that captures both longevity and quality of life, as well as the costs associated with being in a particular health state. ${ }^{26}$ Examples of health states include minor to no neurological disabilities, major neurological disabilities, or death. Health states can be very detailed to capture specific differences between possible outcomes associated with different treatment strategies. For example, health states can capture additional information such as patient age, whether the patient requires additional treatment, or whether the patient is able to return to work and recover income. These models can capture long-term health utilities or costs associated with an intervention and have the potential to quantify the lifelong impact of interventions.

An example of a basic Markov model diagram for a patient with carotid artery stenosis who is offered elective open surgical treatment versus endovascular treatment is provided in Fig. 3. Model inputs populate the Markov model and include the following values: probabilities for transitions in health states, health utilities associated with health states, and costs associated with transitioning to or being in a given health state.

\section{Model Inputs: Transition Probabilities}

Transition probabilities are the probabilities of transitioning to a new health state. ${ }^{27}$ Continuing the example of a patient requiring carotid intervention, if the patient undergoes open surgery, transition probabilities refer to the unique probability values of transition to the new health states of good outcome, poor outcome, or death. In the example illustrated in Fig. 3, sample probabilities associated with clinical outcomes have been provided. Most models from this review (46 studies [84\%]) used other published studies or publicly available information to derive the transition probabilities included in the Markov model. The remaining articles derived model inputs from internal clinical studies in which the probabilities of achieving different functional outcomes were collected. Using either internal clinical studies or publically available information, one can calculate transition probabilities from prior retrospective or prospective clinical data or meta-analyses that capture probabilities of outcomes after interventions. 


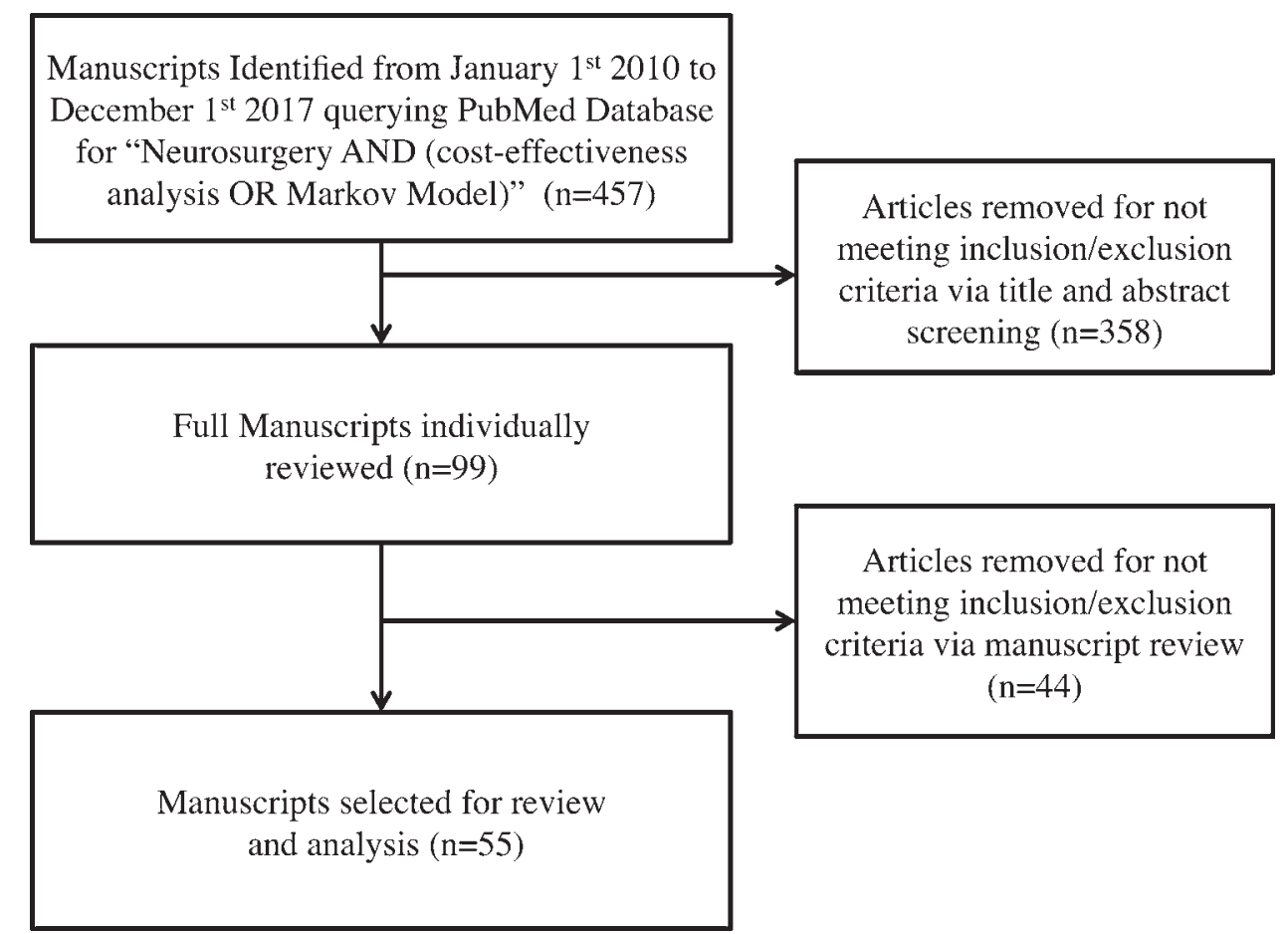

FIG. 1. Flow diagram demonstrating article selection for final analysis. The PubMed database was queried for the dates from January 1, 2010, through December 1, 2017, to identify articles utilizing Markov modeling to perform cost-effectiveness research in neurosurgery. Papers were included in this analysis if 1) Markov modeling was used to conduct cost-effectiveness research and 2) the articles were published in the English language. Articles were excluded if they investigated clinical strategies that were not relevant to neurosurgery.

\section{Model Inputs: Health Utilities}

Health utilities refer to the quality of life value associated with a particular health state. Health utilities are typically measured in quality-adjusted life years (QALYs), which range from 0 (no quality of life or death) to 1 (a full year at full health or quality of life). ${ }^{33}$ Quality-adjusted life years are then accumulated across a period of time such as 1 year, 10 years, or the remainder of a patient's lifetime to quantify the total quality of life and impact on longevity that treatment options may have. Health utilities are typically converted from quality of life questionnaires, such as the SF-36 $36^{3}$ or the EQ-5D, ${ }^{22,30}$ or from other techniques of mapping functional neurological states, such as the modified Rankin Scale score, to a QALY metric. ${ }^{23}$ Continuing the example of a patient with carotid artery disease seeking elective treatment, sample health utilities associated with each state are also provided in Fig. 3. In the recently published neurosurgical literature, quality of life metrics or health utilities associated with each health state were predominantly collected from already published data or publicly available information (44 studies [80\%]). For studies that did not collect health utility information from existing published studies, the investigators conducted surveys such as the SF-36 or EQ-5D as part of a clinical study and converted these values to QALYs.

\section{Model Inputs: Direct and Indirect Costs}

Costs incorporated into Markov models are of two types: direct and indirect. ${ }^{10,15}$ Direct costs are associated with an actual intervention and can include the costs of surgery, anesthesia, hospitalization, imaging, and laboratory tests. Indirect costs refer to costs associated with being in a particular health state such as the costs of rehabilitation, disability, or loss of income. Perspective is an additional characterization of cost information used in Markov modeling that describes the viewpoint of resource expenditure. Cost perspectives are generally divided into two broad groups: 1) societal costs, which refer to aggregated costs sustained by all parties involved with an intervention including direct interventional costs and indirect costs associated with disability, rehabilitation, and lost income; or 2) payer-specific costs, which describe the costs

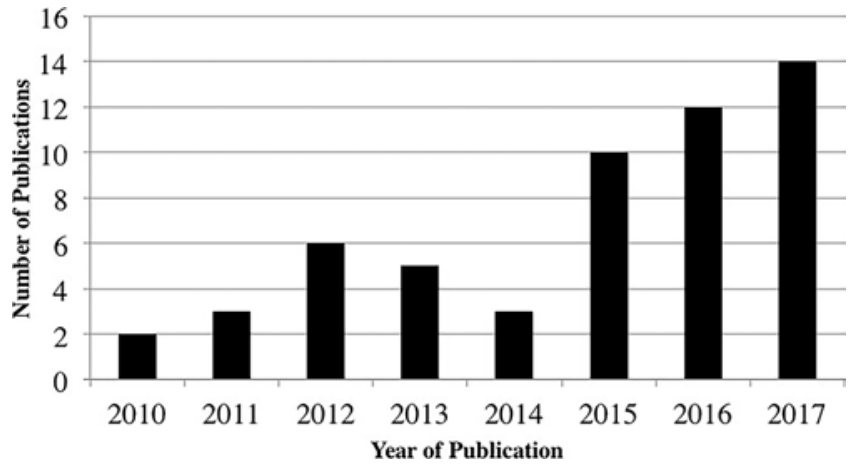

FIG. 2. Neurosurgical publication trends for cost-effectiveness research. 


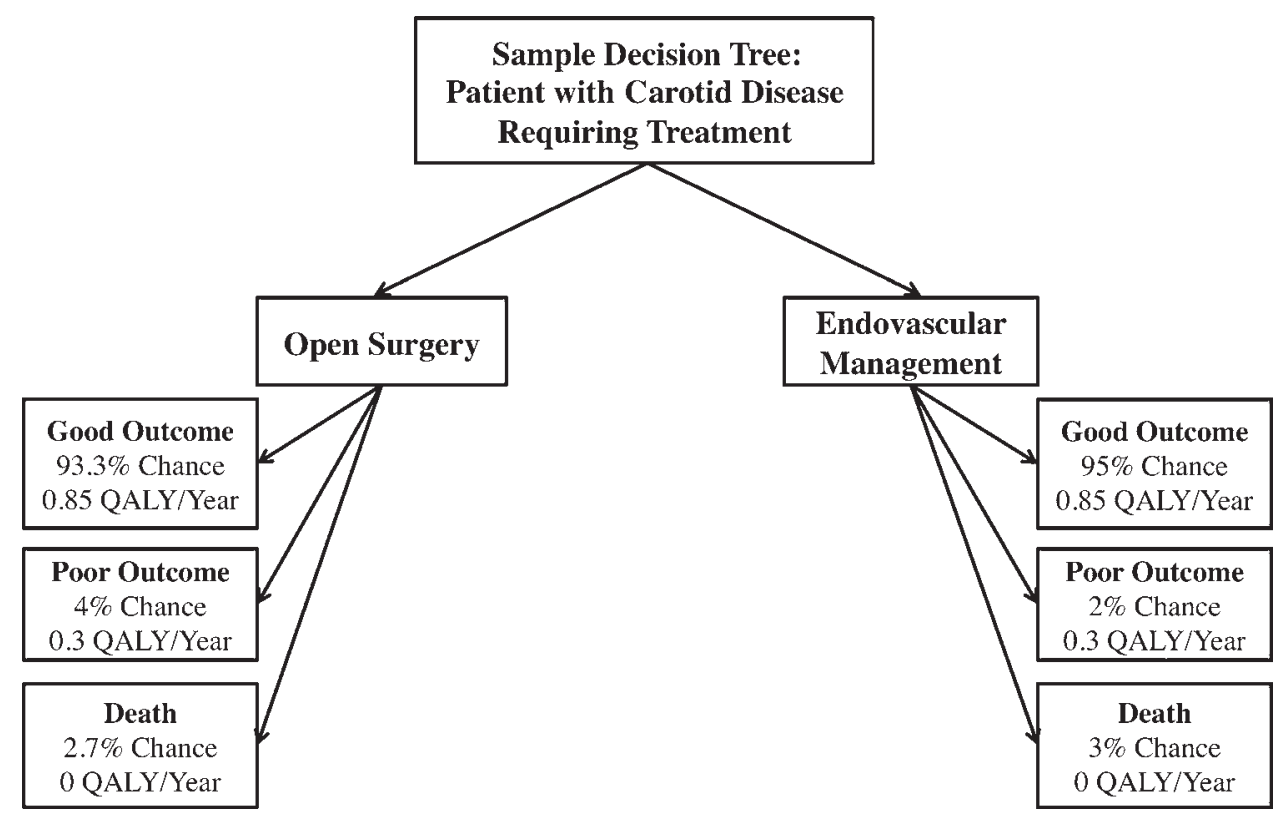

FIG. 3. Illustration of a Markov modeling decision tree that captures the choices available to a patient with carotid artery stenosis who is offered elective treatment. The patient can either undergo open surgery or endovascular management, which will then change the patient's health state from requiring treatment to a good outcome, a poor outcome, or death. Sample probabilities and health utilities associated with each state have been provided.

sustained by an individual, a health insurance company, a third-party payer, or an institution. ${ }^{8,20}$

Of the reviewed articles, $46(84 \%)$ derived direct cost information from published studies or publicly available information, with many (17 articles [40\%]) referring to the Centers for Medicare and Medicaid Services Current Procedural Terminology (CPT) codes. Only 34 papers $(62 \%)$ included indirect costs, $30(88 \%)$ of which derived indirect costs from published studies or publicly available information. The majority of studies $(55 \%)$ utilized a societal perspective for costs, with the remainder using payerspecific costs.

\section{Primary Analytical Techniques: Base Case Analysis and Incremental Cost-Effectiveness Ratio}

All 55 studies identified in this review utilized Markov models to perform a base case analysis and generate an incremental cost-effectiveness ratio (ICER). The base case analysis calculates expected values for total health utilities and total costs associated with each treatment option. All Markov models run for a specified cycle length and number of cycles. Cycle lengths can reflect typical clinical follow-up durations or can be determined by aspects of the research question and can range from weeks to years. For models that last longer than one cycle-such as models that run for the remaining lifespan of the patient facing different treatment strategies-the patient will continue to gain health utilities and incur costs associated with being in that state. Health utilities and costs of future cycles are discounted by a predetermined amount. ${ }^{10}$ Most studies in the literature discount by $3 \%$ for each future year. Discounting future health utilities and costs places a diminished emphasis on future health utilities and costs compared to present health utilities and costs.
The ICER generated from the base case analysis is a ratio of the difference between the total costs of two competing treatment strategies divided by the difference between the total health utilities generated by the two competing treatment strategies. A mathematical representation of the ICER can be described as $(\mathrm{C} 1-\mathrm{C} 0) /(\mathrm{E} 1-\mathrm{E} 0)$, where $(\mathrm{C} 1$ - $\mathrm{C} 0$ ) refers to the difference in costs between two treatment strategies and $(\mathrm{E} 1-\mathrm{E} 0)$ refers to the difference in the health utilities yielded between two treatment strategies. ${ }^{6}$ Treatment strategies are then considered cost-effective if they yield an ICER that is within a predetermined willingness to pay (WTP) threshold. By convention, most costeffectiveness studies place the WTP threshold at \$50,000/ QALY gained or $\$ 100,000 /$ QALY gained. ${ }^{13}$

A sample cost-effectiveness curve generated from a base case analysis in which a patient is selecting between two example interventions (A and B) can be found in Fig. 4. In this sample base case analysis, intervention A yields a total QALY of 16.5 at a total cost of $\$ 76,000$ while intervention B yields a total QALY of 17.1 at a total cost of $\$ 357,700$. This model generates an ICER of $\$ 469,500 /$ QALY gained. At a preset WTP of $\$ 50,000 /$ QALY gained, intervention B would not be considered cost-effective as compared to intervention A. Although intervention B yields more QALYs, it does so at a cost that is not deemed cost-effective at the WTP threshold.

\section{Model Robustness: 1-Way, 2-Way, and Probabilistic Sensitivity Analysis}

While all studies in this review that utilized Markov modeling for cost-effectiveness provided a base case analysis, 51 of them (93\%) conducted 1- or 2-way sensitivity analyses to assess for model robustness, and 37 (67\%) conducted a probabilistic sensitivity analysis (PSA). The 


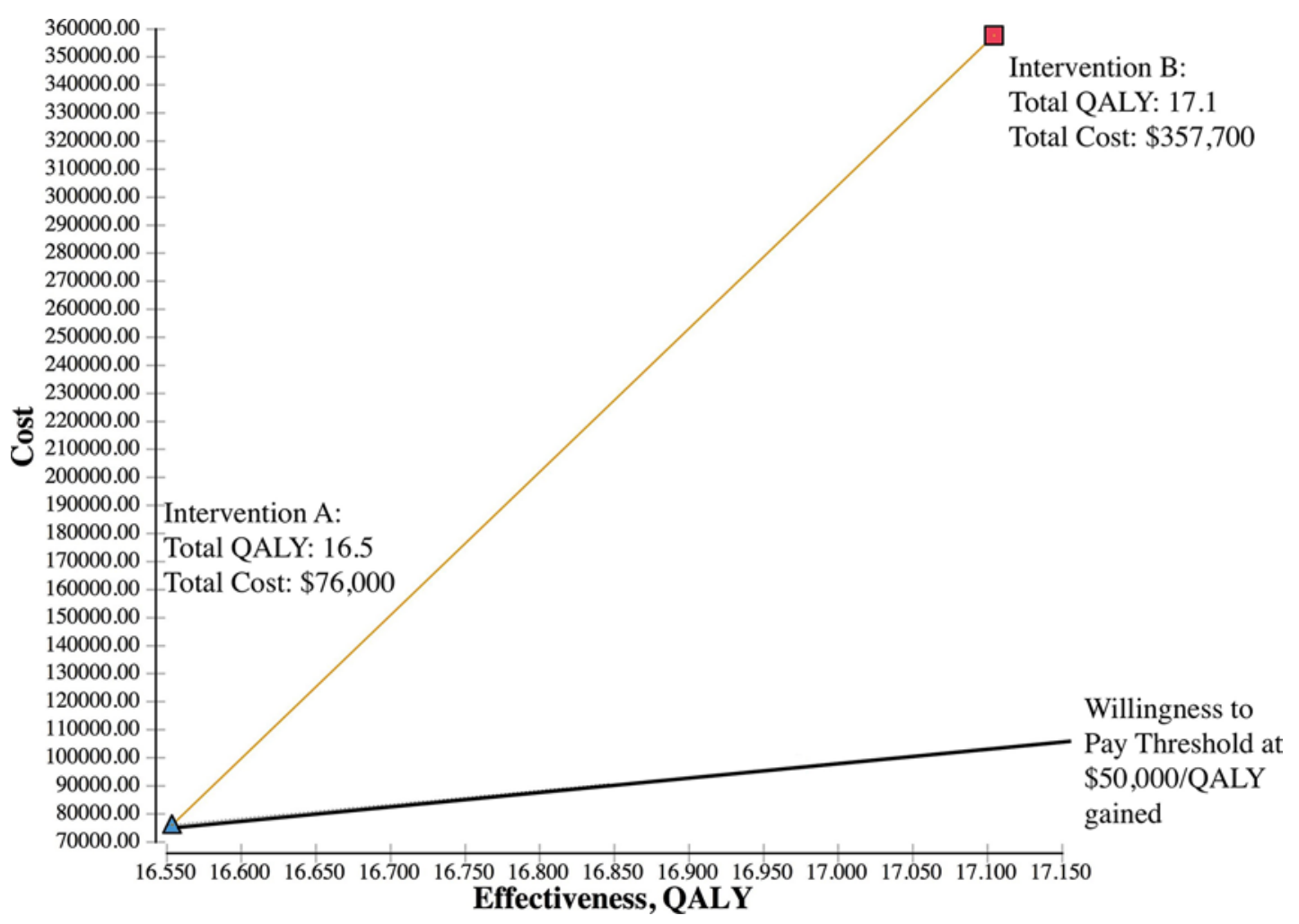

FIG. 4. Illustration of the base case analysis. Intervention A yields a total QALY of 16.5 at a total cost of $\$ 76,000$, whereas intervention B yields a total QALY of 17.1 at a total cost of $\$ 357,700$. This model generates an ICER of $\$ 469,500 / Q A L Y$ gained. At a preset WTP of $\$ 50,000 / Q A L Y$ gained (black line), intervention B would not be considered cost-effective compared to intervention A. Had intervention B yielded total QALYs and costs resulting in a position underneath the black line, this strategy would be considered cost-effective at the WTP threshold of $\$ 50,000 / Q A L Y$ gained.

1-way and 2-way sensitivity analyses improve the robustness of the base case analysis by allowing each model input to range across all values between preset low and high values, as well as investigate how changes in each model input may influence the cost-effectiveness of different treatment options. The 1-way and 2-way sensitivity analyses reveal which input parameters the Markov model is most sensitive to, indicating that certain variable inputs have the strongest influence in determining the most costeffective treatment strategy. The 1-way sensitivity analysis is particularly useful in helping to identify threshold values for a given model input and can demonstrate how the cost-effective strategy may depend on whether or not a model input is greater than or less than an identified threshold value. A clinical example of the 1-way sensitivity analysis can be demonstrated by Wali et al.'s investigation of the elective endovascular treatment of large unruptured aneurysms. ${ }^{35}$ The base case model demonstrated that flow diversion was the cost-effective treatment option for large unruptured aneurysms. The 1-way sensitivity analysis demonstrated that, as the cost of flow diversion ranged from a low value of $\$ 5,000$ to a high value of $\$ 50,000$, flow diversion was no longer cost-effective as compared to coiling when the cost of flow diversion exceeded $\$ 40,100$.

The 2-way sensitivity analysis, by comparison, ranges two model inputs between low and high values simultaneously and may reveal the interplay between how changes in these inputs influence the cost-efficacy of a treatment.
An example 2-way sensitivity analysis can be found in Fig. 5. In this example, costs of interventions A and B that result in a position in the red area would identify intervention B as cost-effective. A position in the blue area would identify intervention A as cost-effective. Continuing the clinical example provided by Wali et al., in investigating elective treatment of large unruptured aneurysms, these authors performed a 2-way sensitivity analysis ranging the costs of elective flow diversion and elective stent-assisted coiling for large aneurysms simultaneously. ${ }^{35}$ In doing so, they could identify specific cost parameters between the two elective interventions to determine how cost-efficacy can change as two model inputs vary simultaneously.

The PSA calculates cost-efficacy influenced by the uncertainty and randomness of each model input using a Monte Carlo simulation. To capture uncertainty and randomness within a Markov model, each model input is assigned an arithmetic mean and standard deviation to capture the range of possible values. This technique can account for distributions in the model values pertaining to costs, surgical success rates, or complication rates to better reflect real life and practice.

A Monte Carlo simulation then runs the model, assigning each model input to a unique value randomly generated from the distribution of values to determine cost-efficacy in a particular iteration of the model. ${ }^{24}$ The Markov model is simulated several thousand times to capture unique iterations in which model inputs are randomly 


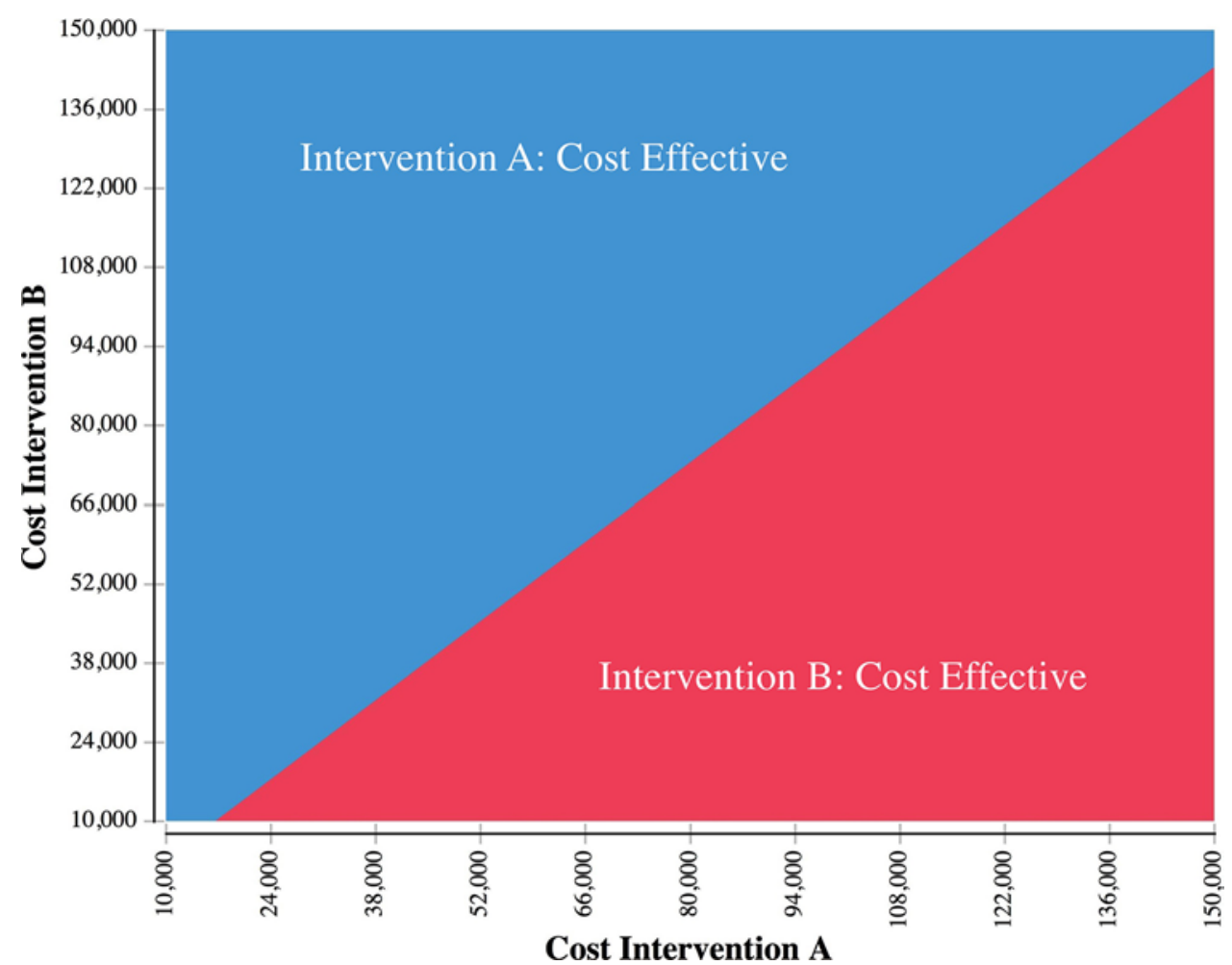

FIG. 5. Sample 2-way sensitivity analysis. As the cost of interventions A and B change across a range of values, the comparative cost-efficacy between the two strategies also changes. The cost coordinates in the red area correspond to model input values that will result in intervention $B$ as more cost-effective, and cost coordinates in the blue area correspond to model input values that will result in intervention $\mathrm{A}$ as more cost-effective.

generated based on the provided mean and standard deviation. Across several thousand iterations, the ICERs yielded from the PSA can converge or consistently demonstrate that one strategy is more cost-effective than another, indicating stability of the cost-effectiveness of one strategy over another despite randomness and change. ${ }^{19}$

An example of a Monte Carlo simulation can be found in the study by Moran et al., who investigated the costeffectiveness of craniotomy for epidural hematomas. ${ }^{18}$ The authors utilized a Monte Carlo simulation across 1000 iterations to capture variability in direct costs associated with surgery, indirect costs associated with lost earning potential, and health utilities associated with each Glasgow Outcome Scale score by providing a mean and standard deviation for each model input. A sample PSA diagram can be found in Fig. 6. In this example PSA, across 10,000 iterations of the Markov model in which each model input had a given mean and standard deviation, intervention A was found to be cost-effective in $58 \%$ of scenarios, while intervention B was found to be cost-effective in $42 \%$ of scenarios, at a WTP threshold of \$50,000/QALY gained.

\section{Discussion}

The United States is in an unprecedented era of health care spending, $, 5,12$ resulting in a national effort to scrutinize the allocation of medical resources and optimize policies that reduce medical waste. ${ }^{2,11}$ Markov modeling and costeffectiveness research contribute to this effort by augment- ing clinical research tools and providing a mathematical basis for determining whether possible gains in longevity or quality of life are cost-effective. This review of the recently published neurosurgical literature demonstrates that neurosurgeons across diverse subspecialties are increasingly publishing work on cost-effective strategies to guide management principles for neurosurgical patients with multiple treatment options.

As the volume of cost-effectiveness literature continues to increase, it is critical for neurosurgeons, even those without formal training in health economics, to be literate in the research methodology in order to better critique and translate pertinent findings to health policy and actual clinical practices. This includes the different analytical techniques, the diverse methods with which model inputs can be obtained and tested, and the limitations and insights of various approaches. The present work reviews the different types of model inputs required to perform cost-effectiveness research and provides a basic framework with which to conceptualize Markov modeling. The majority of the neurosurgical cost-effectiveness research reviewed in the current paper derived model inputs of transition probabilities, health utilities, and health care costs from the published literature or publicly available sources.

There are several potential areas of improvement for increasing the quality of published cost-effectiveness research. As shown in this review, the results of Markov models are directly influenced by model inputs. As more high-powered, prospective, randomized clinical research 


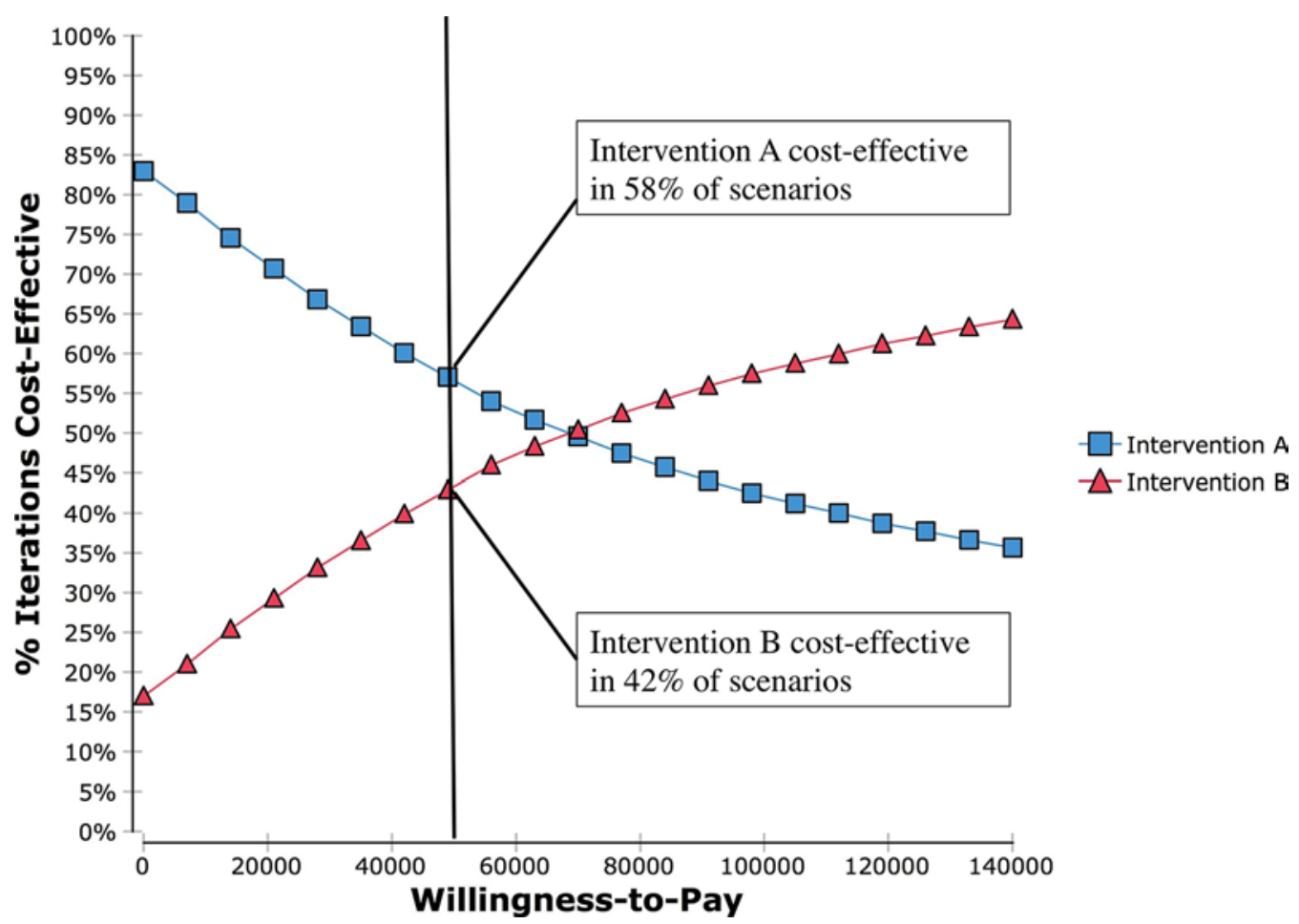

FIG. 6. Sample PSA. Intervention A is cost-effective in $58 \%$ of scenarios, while intervention B is cost-effective in $42 \%$ of scenarios, at a WTP threshold of $\$ 50,000 / Q A L Y$ gained. The vertical black line demarcates the WTP at $\$ 50,000 /$ QALY gained.

studies are published, higher-quality model inputs will be available. With regard to health care costs, only $62 \%$ of the articles in this review included indirect cost information to capture the morbidity, disability, or loss of productivity associated with health states. Subsequent studies can be enhanced by more sophisticated metrics that capture the indirect costs associated with different health states. Regarding Markov model robustness, only 93\% of the studies included in this review augmented the base case analysis with 1-way or 2-way sensitivity analyses, and only $67 \%$ of the models included a PSA-indicating areas for growth to improve the robustness of future Markov models.

As neurosurgeons continue to operate at the intersection of clinical practice, cutting-edge research, and health policy, understanding cost-effectiveness research is essential to remaining at the forefront of health care leadership. Markov modeling is a tool increasingly utilized across diverse neurosurgical subspecialties to investigate the cost-efficacy of novel treatment strategies. We hope that this review of neurosurgical publication patterns and the technical aspects relating to Markov modeling enables the neurosurgical readership to better understand, critique, and apply findings produced from cost-effectiveness research that may have broad implications for health care policy and neurosurgical practice.

\section{Conclusions}

Decision analytic Markov modeling is a powerful tool that enables the neurosurgeon to critically analyze clinical and surgical interventions in terms of optimizing quality of life metrics and the lifelong costs accrued or saved in treating specific neurosurgical pathology. As neurosurgeons continue to innovate and identify novel treatment strategies for patients, Markov modeling will allow for better characterization of the impact of these interventions on both a patient and societal level.

\section{Acknowledgments}

The project described was partially supported by the National Institutes of Health Pre-doctoral Grant No. TL1TR001443 (M.G.B.).

\section{References}

1. Ament JD, Kim KD: Standardizing cost-utility analysis in neurosurgery. Neurosurg Focus 33(1):E4, 2012

2. Berwick DM, Hackbarth AD: Eliminating waste in US health care. JAMA 307:1513-1516, 2012

3. Brazier J, Roberts J, Deverill M: The estimation of a preference-based measure of health from the SF-36. J Health Econ 21:271-292, 2002

4. Briggs A, Sculpher M: An introduction to Markov modelling for economic evaluation. Pharmacoeconomics 13:397-409, 1998

5. Chen A, Goldman D: Health care spending: historical trends and new directions. Annu Rev Econ 8:291-319, 2016

6. Craig BA, Black MA: Incremental cost-effectiveness ratio and incremental net-health benefit: two sides of the same coin. Expert Rev Pharmacoecon Outcomes Res 1:37-46, 2001

7. Doubilet P, Begg CB, Weinstein MC, Braun P, McNeil BJ: Probabilistic sensitivity analysis using Monte Carlo simulation. A practical approach. Med Decis Making 5:157-177, 1985

8. Drummond MF, Sculpher MJ, Claxton K, Stoddart GL, Torrance GW: Methods for the Economic Evaluation of 
Health Care Programmes, ed 4. New York: Oxford University Press, 2015

9. Finkelstein EA, Trogdon JG, Cohen JW, Dietz W: Annual medical spending attributable to obesity: payer-and servicespecific estimates. Health Aff (Millwood) 28:w822-w831, 2009

10. Gold MR, Siegel JE, Russell LB, Weinstein MC (eds): CostEffectiveness in Health and Medicine. New York: Oxford University Press, 1996

11. Grosse SD, Teutsch SM, Haddix AC: Lessons from costeffectiveness research for United States public health policy. Annu Rev Public Health 28:365-391, 2007

12. Hartman M, Martin AB, Espinosa N, Catlin A: National health care spending in 2016: spending and enrollment growth slow after initial coverage expansions. Health Aff (Millwood) 37:150-160, 2018

13. Hirth RA, Chernew ME, Miller E, Fendrick AM, Weissert WG: Willingness to pay for a quality-adjusted life year: in search of a standard. Med Decis Making 20:332-342, 2000

14. Hoffman C, Rice D, Sung HY: Persons with chronic conditions. Their prevalence and costs. JAMA 276:1473-1479, 1996

15. Koopmanschap MA, Rutten FF: Indirect costs in economic studies: confronting the confusion. Pharmacoeconomics 4:446-454, 1993

16. Lakdawalla DN, Bhattacharya J, Goldman DP: Are the young becoming more disabled? Health Aff (Millwood) 23:168 176,2004

17. McLaughlin N, Ong MK, Tabbush V, Hagigi F, Martin NA: Contemporary health care economics: an overview. Neurosurg Focus 37(5):E2, 2014

18. Moran D, Shrime MG, Nang S, Vycheth I, Vuthy D, Hong R, et al: Cost-effectiveness of craniotomy for epidural hematomas at a major government hospital in Cambodia. World J Surg 41:2215-2223, 2017

19. Munkin MK, Trivedi PK: Bayesian analysis of a self-selection model with multiple outcomes using simulation-based estimation: an application to the demand for healthcare. $\mathbf{J}$ Econom 114:197-220, 2003

20. Neumann PJ: Costing and perspective in published costeffectiveness analysis. Med Care 47 (7 Suppl 1):S28-S32, 2009

21. Pearson SD: Cost, coverage, and comparative effectiveness research: the critical issues for oncology. J Clin Oncol 30:4275-4281, 2012

22. Ramos-Goñi JM, Rivero-Arias O: eq5d: A command to calculate index values for the EQ-5D quality-of-life instrument. Stata J 11:120-125, 2011

23. Rivero-Arias O, Ouellet M, Gray A, Wolstenholme J, Rothwell PM, Luengo-Fernandez R: Mapping the modified Rankin scale (mRS) measurement into the generic EuroQol (EQ-5D) health outcome. Med Decis Making 30:341-354, 2010

24. Rubinstein RY, Kroese DP: Simulation and the Monte Carlo Method. Hoboken: Wiley, 2016, Vol 10

25. Schneider EL, Guralnik JM: The aging of America. Impact on health care costs. JAMA 263:2335-2340, 1990

26. Sculpher M, Fenwick E, Claxton K: Assessing quality in decision analytic cost-effectiveness models. A suggested framework and example of application. Pharmacoeconomics 17:461-477, 2000

27. Sonnenberg FA, Beck JR: Markov models in medical decision making: a practical guide. Med Decis Making 13:322338, 1993

28. Stein SC: Comparative effectiveness in neurosurgery: what it means, how it is measured, and why it matters. Neurosurg Focus 33(1):E1, 2012

29. Stein SC, Burnett MG: Decision analysis to estimate cost effectiveness in neurosurgery. Neurosurg Focus 12(4):e5, 2002

30. Szende A, Oppe M, Devlin N: EQ-5D Value Sets: Inventory, Comparative Review and User Guide. Dordrecht: Springer, 2007, Vol 2

31. Thoma A, Strumas N, Rockwell G, McKnight L: The use of cost-effectiveness analysis in plastic surgery clinical research. Clin Plast Surg 35:285-296, 2008

32. Thorpe KE, Florence CS, Howard DH, Joski P: The impact of obesity on rising medical spending. Health Aff (Millwood) 23 (Suppl Web Exclusives):W4-480-W4-486, 2004

33. Torrance GW, Feeny D: Utilities and quality-adjusted life years. Int J Technol Assess Health Care 5:559-575, 1989

34. Wali AR, Park CC, Brown JM, Mandeville R: Analyzing cost-effectiveness of ulnar and median nerve transfers to regain forearm flexion. Neurosurg Focus 42(3):E11, 2017

35. Wali AR, Park CC, Santiago-Dieppa DR, Vaida F, Murphy JD, Khalessi AA: Pipeline embolization device versus coiling for the treatment of large and giant unruptured intracranial aneurysms: a cost-effectiveness analysis. Neurosurg Focus 42(6):E6, 2017

36. Wali AR, Santiago-Dieppa DR, Brown JM, Mandeville R: Nerve transfer versus muscle transfer to restore elbow flexion after pan-brachial plexus injury: a cost-effectiveness analysis. Neurosurg Focus 43(1):E4, 2017

37. Weinstein MC: Principles of cost-effective resource allocation in health care organizations. Int J Technol Assess Health Care 6:93-103, 1990

\section{Disclosures}

The authors report no conflict of interest concerning the materials or methods used in this study or the findings specified in this paper.

\section{Author Contributions}

Conception and design: Wali, Brandel, Santiago-Dieppa, Rennert. Acquisition of data: Wali. Analysis and interpretation of data: Wali, Murphy. Drafting the article: Wali, Brandel, SantiagoDieppa, Rennert, Steinberg, Hirshman. Critically revising the article: all authors. Reviewed submitted version of manuscript: all authors. Study supervision: Khalessi.

\section{Correspondence}

Alexander A. Khalessi: University of California, San Diego, CA. akhalessi@ucsd.edu. 